\title{
Comparative Study of the Rhizosphere and Root Endosphere Microbiomes of Cholistan Desert Plants
}

\author{
Salma Mukhtar*, Samina Mehnaz and Kauser Abdulla Malik* \\ KAM School of Life Sciences, Forman Christian College (A Chartered University), Lahore, Pakistan
}

Microbial communities associated with the rhizosphere and roots of desert halophytes play an important role in plants' growth and development. Very limited information has been available on the microbial diversity of arid environments of Pakistan. Hence in the current study, the microbial diversity of rhizosphere and root endosphere of desert halophytes, Zygophyllum simplex, Haloxylon salicoricum, Aerva javanica, and Capparis decidua was evaluated. The rhizosphere and root endosphere samples of desert halophytes collected from the three geographic sites of Cholistan desert, Punjab, Pakistan were analyzed by using 16S rRNA based Illumina sequencing. The results showed that Proteobacteria were more abundant in the

Edited by:

Philippe Vandenkoornhuyse, University of Rennes 1, France

Reviewed by:

Ajar Nath Yadav,

Eternal University, India $\mathrm{Jie} \mathrm{Hu}$,

University of Rennes 1, France

*Correspondence:

Kauser Abdulla Malik

kausermalik@fccollege.edu.pk

Salma Mukhtar

salmamukhtar85@gmail.com

Specialty section:

This article was submitted to

Terrestrial Microbiology,

a section of the journal

Frontiers in Microbiology

Received: 18 October 2020

Accepted: 04 March 2021

Published: 26 March 2021

Citation:

Mukhtar S, Mehnaz S and Malik KA (2021) Comparative Study

of the Rhizosphere and Root

Endosphere Microbiomes

of Cholistan Desert Plants.

Front. Microbiol. 12:618742.

doi: 10.3389/fmicb.2021.618742 rhizospheric soils while Actinobacteria were more dominant in the root endosphere of halophytes. Bacteroidetes, Firmicutes, and Deinococcus-Thermus were identified from all rhizospheric soils and roots across the three sites, with variable percentage. Bacillus, Kocuria, Pseudomonas, Halomonas, and Flavobacterium were commonly identified from the rhizosphere and root endosphere of halophytes across all the three sites. At the genus level, microbial diversity from Haloxylon showed the greatest variations between the rhizosphere and root endosphere from the site 2 . This study revealed that microbial diversity analysis can be used to study how changes in abiotic factors such as soil moisture content and salinity affect the microbial communities associated with the rhizospheric soils and root endosphere of halophytes across the three sites. This study will also help in the discovery of potential inoculants for crops growing in arid and semi-arid regions of Pakistan.

Keywords: halophytes, root endosphere, Illumina sequencing, 16S rRNA gene, halophilic bacteria

\section{INTRODUCTION}

Soil salinity is considered as one of the major abiotic pressures that affects more than 840 million hectares of agricultural area worldwide. Soil salinity continuously increase in arid and semiarid regions globally. Salinization reduces plant growth and adversely affects the crops yield and productivity (Rengasamy, 2006; Etesami and Beattie, 2018). Halophytes growing in arid and saline environments play a vital role in the maintenance of soil composition by nutrient mineralization and cycling, sequestration of carbon and improving the micro-environments (English and Colmer, 2011). These plants have great potential to preserve ecosystems. They can be used for production of biofuel and fiber and as fodder crops (Dagla and Shekhawat, 2005; Chaudhary et al., 2015). 
Plant microbiome serves as a "second genome" to the plant and plays an important role in growth and productivity of halophytes. Rhizosphere and root endosphere microbiomes from extreme environments such as arid, saline, hot, cold, and acidic help the plants to grow in these environments (Oren, 2013; Santhanam et al., 2017; Mukhtar et al., 2018). Recent studies about rhizosphere microbiome of desert halophytes revealed a high portion of halophilic bacteria as compared to rhizosphere microbiome of salinity sensitive plants (Marasco et al., 2016; Tian and Zhang, 2017). A number of halophilic bacterial genera including Bacillus, Halomonas, Halobacillus, Oceanobacillus, Marinobacter, Marinococcus, and Nesterenkonia have been identified from the rhizosphere and roots of halophytes and xerophytes (Ramadoss et al., 2013; Zhao et al., 2016; Etesami and Maheshwari, 2018; Mukhtar et al., 2019).

It is well documented that halophilic and halotolerant PGPRs (plant growth promoting bacteria) enhance growth of halophytes (Yuan et al., 2016; Mukhtar et al., 2017a). These bacteria have the ability to improve plant growth and ultimately increase crop yield by nitrogen fixation, solubilization of minerals, production of phytohormones and siderophores, under salinity and drought conditions (Hussain et al., 2015; Zhou et al., 2017; Etesami and Beattie, 2018). PGPRs also provide plant protection against fungal and bacterial diseases by production of a variety of antifungal and antibacterial compounds (Bulgarelli et al., 2013). Halophiles are able to produce different industrially important enzymes such as protease, lipase, amylase, laccase, xylanase, and cellulase with polyextremophilic properties (Kumar et al., 2012; Mukhtar et al., 2019). Halophilic enzymes have potential for different industrial applications including textile, paper and pulp, pharmaceutical, baking, and detergent industries (Liszka et al., 2012; Mukhtar et al., 2019). Halophilic bacteria can also be used for bioremediation of a variety of hazardous compounds in saline and arid environments (Liszka et al., 2012; Castillo-Carvajal et al., 2014; Tian and Gao, 2014).

A number of studies have already reported desert halophytes and xerophytes, such as Cactus, Agave, Halocnemum, Halostachys, Lycium, Salicornia, and Kalidium associated microbial diversity by using high throughput sequencing approaches (Coleman-Derr et al., 2016; Fonseca-Garcia et al., 2016; Yuan et al., 2016; Tian and Zhang, 2017). However, the microbial communities associated with the desert halophytes growing in Cholistan, Pakistan, an extremely dry and saline environment, have not been previously explored. Authors evaluated the rhizosphere and root endosphere microbiomes of four desert halophytes Zygophyllum simplex, Haloxylon salicoricum, Aerva javanica, and Capparis decidua, collected from three sites by using culture-independent (Illumina sequencing) approaches. The main objectives of the present study were: (1) to compare the microbial communities associated with the rhizosphere and root endosphere of each halophyte, individually, among the three geographic sites and (2) to compare the microbial diversity from the rhizosphere and root endosphere of four halophyte species so as to understand the differences among the plant species and across the three geographic sites.

\section{MATERIALS AND METHODS}

\section{Soil Sampling}

Cholistan is a hot arid sandy desert covering an area of $26,000 \mathrm{~km}^{2}$ and is locally known as Rohi. It is situated in the South-West of Punjab province (Pakistan) and has an average annual rainfall from 128 to $178 \mathrm{~mm}$ (Supplementary Figure 1). Geographically, it is located $27^{\circ} 42^{\prime}$ and $29^{\circ} 45^{\prime}$ North, $69^{\circ} 52^{\prime}$ and $75^{\circ} 24^{\prime}$ East. Water is available at $25-90 \mathrm{~m}$ depth and is too brackish. Drought in this region is quite common, sometimes extending from 2 to 3 years, causing a lot of harms (Chaudhry and Nasim, 1995). Vegetation in this area includes a variety of grasses (Panicum, Cenchrus, Aristida, and Lasiurus), herbs (Suaeda, Chenopodium, Aerva, Zygophyllum, and Dipterygium), and shrubs (Haloxylon, Justicia, Capparis). Authors have surveyed an area of approximately $71 \mathrm{~km}$ near the Lal Suhanra National Park, Cholistan, and selected three geographic sites (approximately $23 \mathrm{~km}$ far from each other) for sampling of halophytes according to land use and vegetation cover (Supplementary Figure 2). These sampling sites were considered as the most drought affected regions. Drought tolerant halophytes, including Zygophyllum simplex, Haloxylon salicoricum, Aerva javanica, and Capparis decidua, dominant across all three sites, were collected. The sampling area was selected according to land use and vegetation cover. The whole plants were excavated with surrounding soil in blocks $(15-20 \mathrm{~cm}$ in depth, $20 \mathrm{~cm}$ in width, and $20 \mathrm{~cm}$ in length). Four replicates for each plant and soil sample were collected from each site (Koranda et al., 2011; Mukhtar et al., 2018). Plant and soil samples were taken to the laboratory from the collection site in an ice box and stored at $-80^{\circ} \mathrm{C}$ for microbial diversity analysis.

\section{Soil Physicochemical Characteristics}

About 350 grams of soil were dried and sieved for estimation of physicochemical properties. The $\mathrm{pH}$ of soil was measured by preparing a mixture of 1:2.5 (w/v) soil to water. Electrical conductivity $(\mathrm{dS} / \mathrm{m})$ was calculated according to Adviento-Borbe et al. (2006). Soil moisture and texture class were estimated by using the method from Anderson and Ingram (1993). Total organic carbon $\left(\mathrm{C}_{\text {org }}\right)$ was determined by Walkley and Black (1934) method (1934) and total organic nitrogen was calculated using Kjeldahl method. Available phosphorous (P) was estimated by using a calorimetric method with sodium bicarbonate, ammonium molybdate, and ascorbic acid (Olsen et al., 1954). Potassium (K), calcium (Ca), and magnesium (Mg) were detected by atomic absorption spectrometry. Carbonate $\left(\mathrm{CO}_{3}{ }^{2-}\right)$ and bicarbonate $\left(\mathrm{HCO}_{3}{ }^{-}\right)$ions were determined by using Vogel (1978) method (1978).

\section{DNA Extraction, Amplification of $16 \mathrm{~S}$ rRNA Gene and Illumina MiSeq Sequencing}

About $100 \mathrm{~g}$ of rhizospheric soil was mixed thoroughly and filtered with $2 \mathrm{~mm}$ sieve. The total DNA from soil samples $(0.5 \mathrm{~g})$ were extracted using FastDNA Spin Kit for Soil, according to manufacturer's instructions. For DNA extraction from root 
endosphere, roots were surface sterilized according to Mukhtar et al. (2019) and FastDNA Spin Kit specific for plant tissues was used. The quality of DNA was determined by using $0.9 \%$ agarose gel and quantity was measured by using Nanodrop (NanoDrop 200c Thermo Fisher Scientific, Waltham, MA, United States).

In total, 96 DNA samples; 12 rhizosphere and 12 root samples of each plant collected from the three geographic sites were used for amplification of 16S rRNA gene and Illumina (MiSeq) sequencing. The V3-V4 region of the 16S rRNA gene was amplified by using primers (Bakt_341F: CCTACGGGNGGCWGCAG and Bakt_805R: GACTACHVGGGTATCTAATCC), which were linked with unique identifier and adapter sequences (Supplementary Table 1). The detailed PCR conditions for amplicon sequencing were the same as described by Herlemann et al. (2011). Amplified PCR products were purified with Agencourt AMPure beads (Beckman Coulter, Brea, CA, United States). Finally, about $10 \mathrm{ng}$ of DNA from each sample was sequenced on the Illumina MiSeq platform by Macrogen (Geumcheon-gu, Seoul, South Korea).

\section{Bioinformatics and Statistical Analyses}

Sequences were processed and sorted using the default parameters in QIIME 1.3 (Caporaso et al., 2010). An offset of 10 nucleotides was set in order to remove the first 10 bases of each sequence, and high quality sequences with an average length of 350 bases were selected. Chimera Slayer software was used to check chimeric sequences (DeSantis et al., 2006). De novo OTU (operational taxonomic unit) picking was done to generate OTU files using UCLUST that is a default parameter of QIIME, with 97\% sequence similarity and RDP classifier was used to assign taxonomy (Wang et al., 2007). For different taxonomic levels, such as phylum, class, order, family, and genus, Good's coverage was calculated by using 97\% similarity cutoff (Good, 1953).

Alpha and beta diversity were determined using QIIME alpha_rerefaction.py and beta_diversity_through_plots.py commands, respectively. Alpha diversity was calculated at a sequence depth of 82,152 reads per soil sample, as alpha diversity indices are correlated with the number of sequences by using the Kruskal-Wallis test. The selected maximum sampling depth corresponded to minimum number of reads obtained from any of the remaining sequenced samples. Beta diversity was analyzed by using non-metric multidimensional scaling analysis (NMDS) in "Mass" and "vegan" packages. A matrix was calculated using the weighted and unweighted UniFrac distances among samples at a sequence depth of 82,152 reads per soil sample (Lozupone et al., 2011). Distances were calculated using the "envfit" function of the package "vegan" for Bray-Curtis. To further explore the relationship between bacterial communities and soil properties, redundancy analysis (RDA) and mental test were performed to study the relationship between the most abundant bacteria and soil properties. To explain the differences in the composition of taxa inside the data matrix community, a heatmap (relative abundance matrix) was generated at class level using XLSTAT 7.0 software (Fahmy, 2003). Number of OTUs was calculated by using Venn diagrams analysis and distances were calculated using the "vegdist" function of the "vegan R package." Variation partitioning was performed on the basis of plant species, soil physicochemical characteristics, and geographical distance on composition of rhizosphere and root endosphere microbiomes across the three sites.

To detect the taxonomic classifications that were significantly abundant in rhizospheric soils and root samples, Wilkcoxon's non-parametric rank-sum test and LDA using the LEfSe (LDA Effective Size) program was used (Segata et al., 2011). One-way ANOVA was applied to analyze differences among rhizospheric soils and root samples. Environmental fitting analysis using "envfit" function in vegan $\mathrm{R}$ package was used to find out which soil physicochemical factors strongly associated with rhizosphere and root endosphere microbiomes. To predict the functional profile of bacteria identified from the rhizosphere and root endosphere of halophytes, Tax4Fun software ( $\mathrm{R}$ package) was used (Abhauer et al., 2015). Variation partitioning analysis was performed to study the impact of the relative influences of plant species, soil physicochemical characteristics, and geographical distance on composition of rhizosphere and root endosphere microbiomes. Retrieved 16S rRNA sequences datasets were deposited in NCBI GenBank under SRA accession numbers SRR9588854 to SRR9588861.

\section{RESULTS}

\section{Correlations Between Physicochemical Characteristics of Soil and Microbial Community Structure}

Soil $\mathrm{pH}$ ranged from 7.02 to 7.69 with the maximum value in Haloxylon soils collected from the site 2 and the minimum in Zygophyllum soils collected from the site (Table 1), electrical conductance $\left(\mathrm{EC}_{1: 1}\right)$ ranged from 3.79 to $5.51 \mathrm{dS} / \mathrm{m}$ with the maximum value in Haloxylon soils collected from the site 2 and minimum in Aerva soils collected from the site 3 (Table 1). The moisture content ranged from 20.25 to $25.49 \%$. The soil samples collected from site 2 were more dried as compared to soil samples from the geographic sites 1 and 3 . Soil temperature ranged from 38.79 to $42.54^{\circ} \mathrm{C}$ (Table 1). The average organic matter ranged from 12.55 to $18.37 \mathrm{~g} \cdot \mathrm{kg}^{-1}$ with the highest values in Capparis rhizospheric soils collected from site 2 and the lowest in Haloxylon rhizospheric soils collected from site 1 . The average values for $\mathrm{P}, \mathrm{K}, \mathrm{Ca}$, and $\mathrm{Mg}$ contents were 6.78, 0.55, 182.25, and $82.16 \mathrm{mg} . \mathrm{kg}^{-1}$, respectively. The values of nitrate ions were higher in Capparis rhizospheric soils as compared to other plants soils from all sites. Carbonate and bicarbonate ions were higher in Haloxylon and Aerva rhizospheric soils, as compared to other plants soils (Table 1).

Differences in community structure among different rhizospheric soil and root samples across the three geographic sites were explained by redundancy analysis (RDA) (Figure 1). It was observed that microbial communities in the rhizosphere and roots of Haloxylon and Zygophyllum showed greatest variations as compared to microbial communities identified 


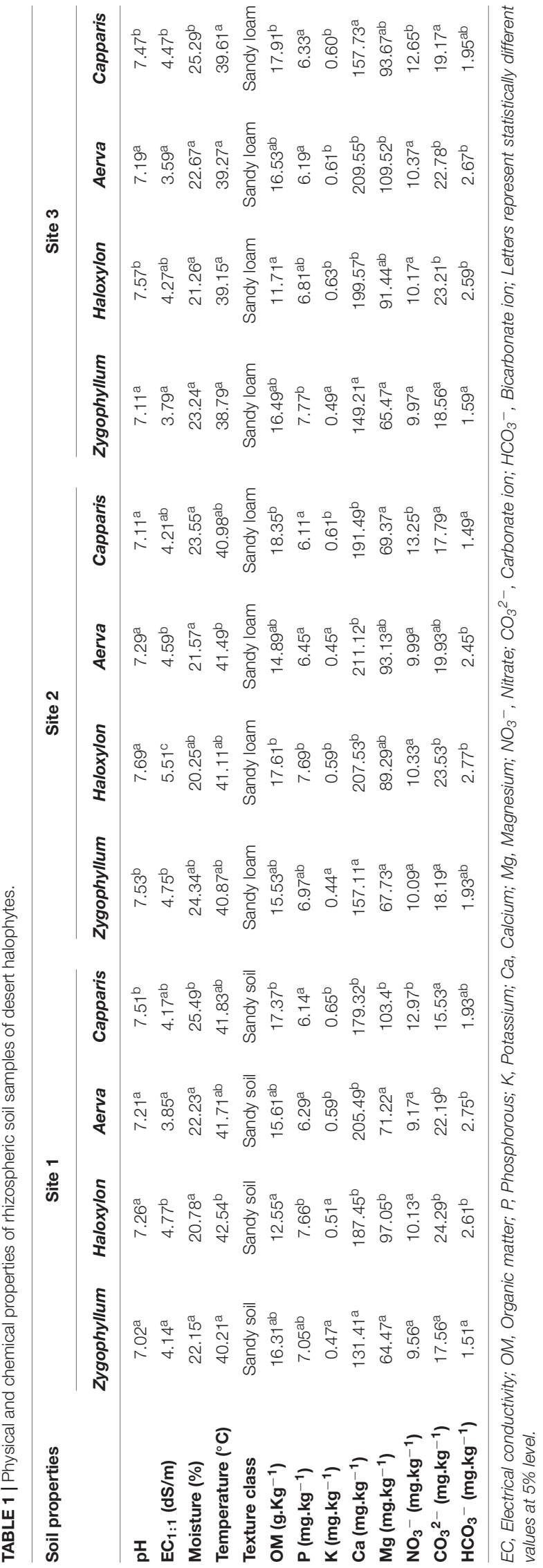

from the rhizosphere and roots of Aerva and Capparis, from the geographic site 2 (Figure 1). Some bacterial genera were more abundant than others at each sampling site. Soil physicochemical characteristics, especially salinity and moisture content affect the microbial diversity in each plant across the three sites.

\section{General Characteristics of 16S rRNA Based Illumina Sequencing}

In this study, a total of 697743 sequences were obtained from the rhizospheric soil samples and 647802 sequences from the root endosphere of halophytes, before the removal of mitochondrial and plastid contaminants while 638292 sequences were obtained from the rhizospheric soil samples and 530006 sequences from the root endosphere of halophytes, after the removal of mitochondrial and plastid contaminants (Supplementary Table 2).

About $67-83 \%$ reads were assigned at the phylum level, $59-81 \%$ to class level, $34-51 \%$ to the family, and $31-49 \%$ were assigned to the genus level, from the geographic site 1 (Supplementary Figure 3). About 71-81\% reads were assigned at the phylum level, $51-72 \%$ to the class level, $34-51 \%$ to the family, and $27-50 \%$ were assigned to the genus level, from the geographic site 2 (Supplementary Figure 4). About $69-81 \%$ reads were assigned at the phylum level, $57-71 \%$ to the class level, $29-55 \%$ to the family, and $26-45 \%$ were assigned to the genus level from the geographic site 3 (Supplementary Figure 5).

\section{Microbial Diversity Comparisons at Global OTU Level}

The results for observed OTUs for microbial communities identified from the rhizosphere and root endosphere of Haloxylon were highly variable as compared to soil and root samples of other plants, collected across all sites (Figure 2A and Supplementary Table 3). Within plant species, alpha diversity was highest in the rhizosphere and root endosphere of Haloxylon from the geographic site 2. Overall, microbial communities from the rhizosphere of all plants showed higher diversity as compared to the root endosphere and microbial diversity differed significantly among plant species and across the three sites (Figure 2B and Supplementary Table 4).

Beta diversity was calculated by using weighted and unweighted UniFrac distances for non-metric multidimensional scaling plots. Microbial communities associated with the rhizosphere and root endosphere were clustered significantly different among the plant species and across the sites (Figures $3 \mathbf{A}, \mathbf{B})$. In case of site 1 , microbial diversity from the rhizosphere samples was clustered differently from the root endosphere samples, within and between plant species (Figures 3C,D). The microbial communities identified from the site 2 showed higher variations among plant-type and sample-type as compared to microbial communities identified from the site 1 and site 3 (Figures $3 \mathrm{E}-\mathrm{H}$, respectively). Overall, the NMDS analysis showed that the rhizosphere and root endosphere microbiomes of Haloxylon exhibited the highest variations from the site 2 . 


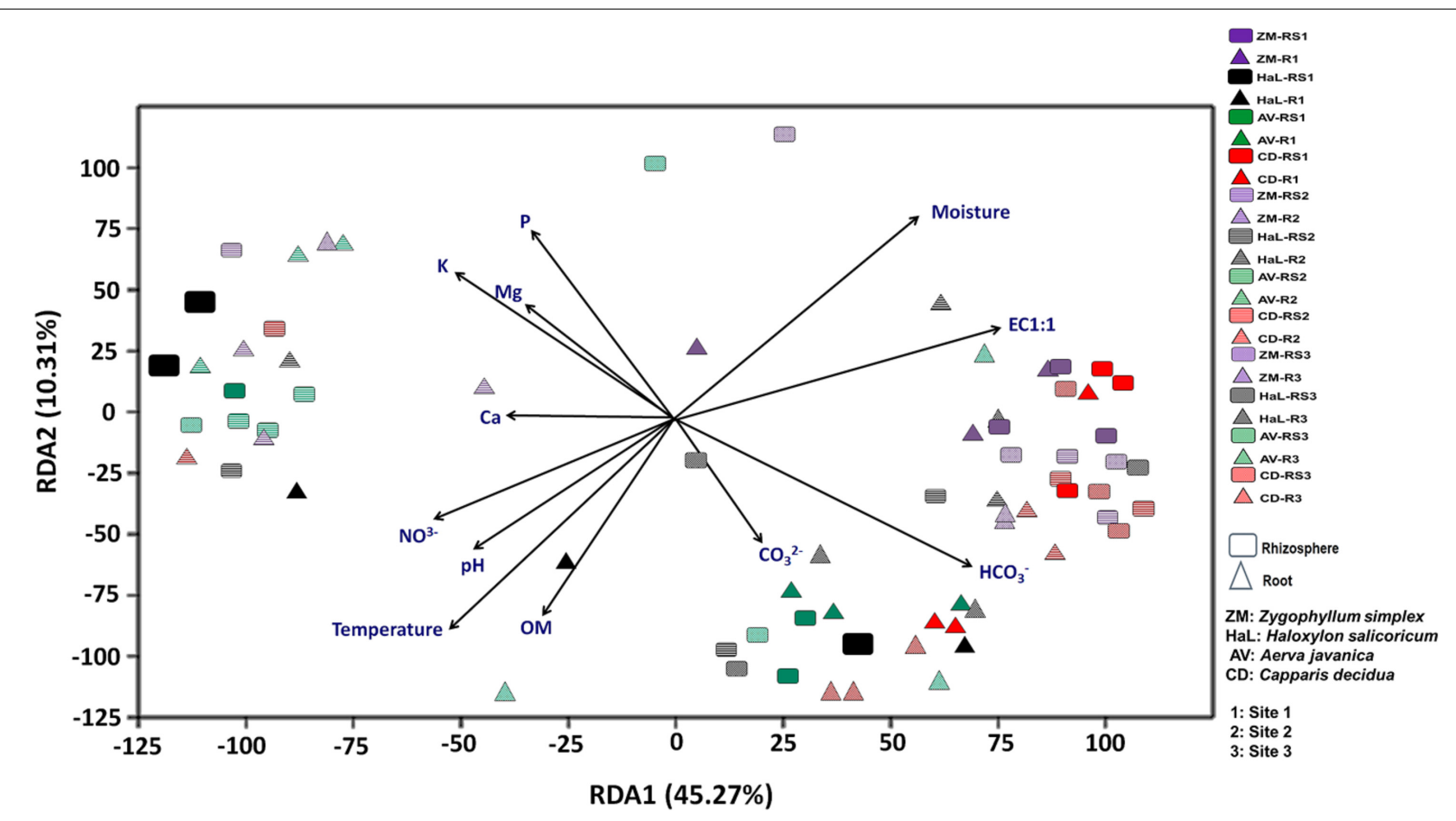

FIGURE 1 | Environmental fitting analysis of rhizosphere and root endosphere microbiomes across the three sites by using redundancy analysis (RDA) plot.
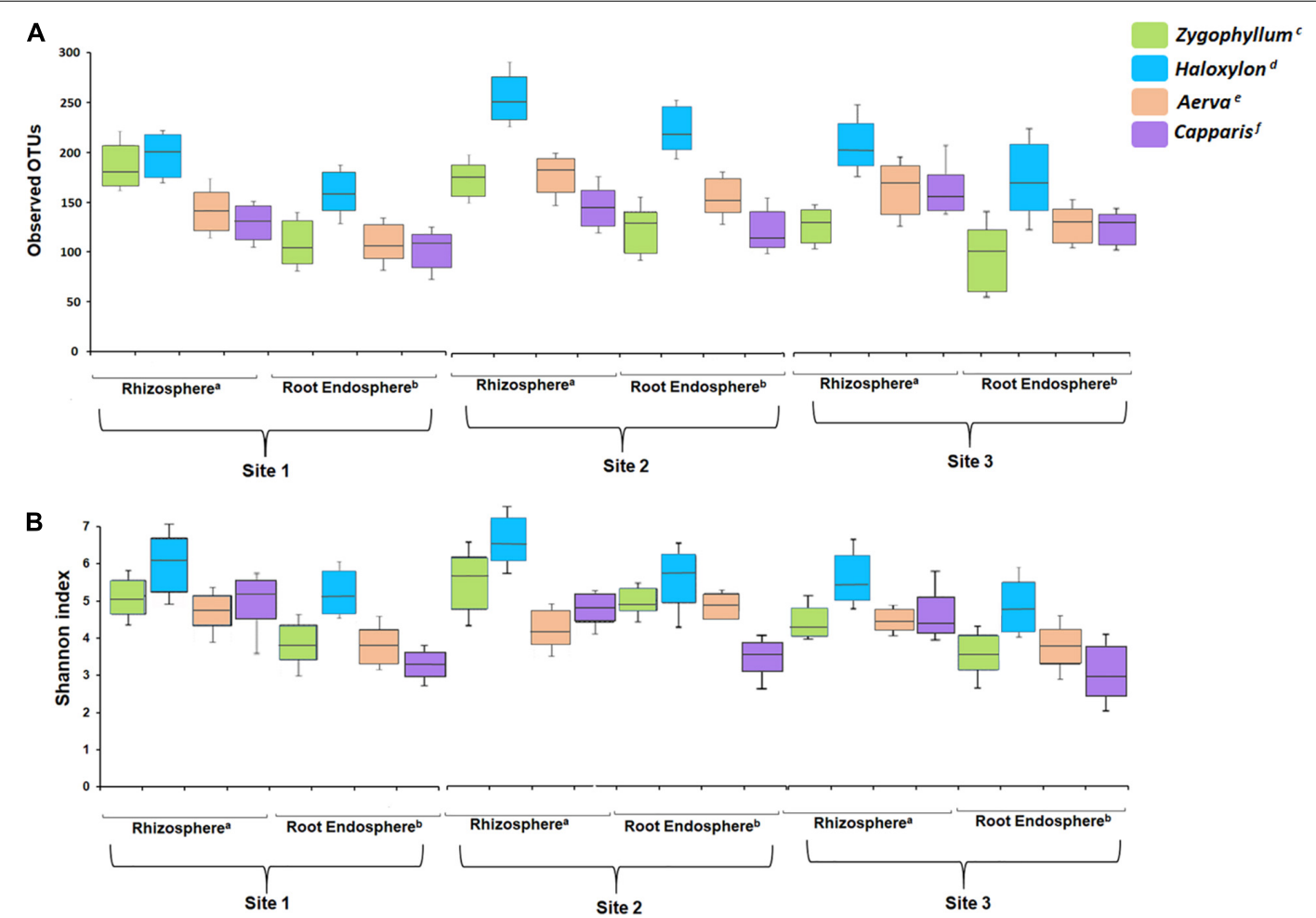

FIGURE 2 | Alpha diversity analysis. (A) Number of observed OTUs and (B) Shannon index. All the soil and root samples showed significant difference after a Kruskal-Wallis test with a confidence level of $99 \%(p \leq 0.05)$ in each index. Superscripts $(a$ and $b)$ indicate significant differences in the rhizosphere and root endosphere among plant species, while superscripts (c-f) indicate significant differences between sample types associated with a plant species. 

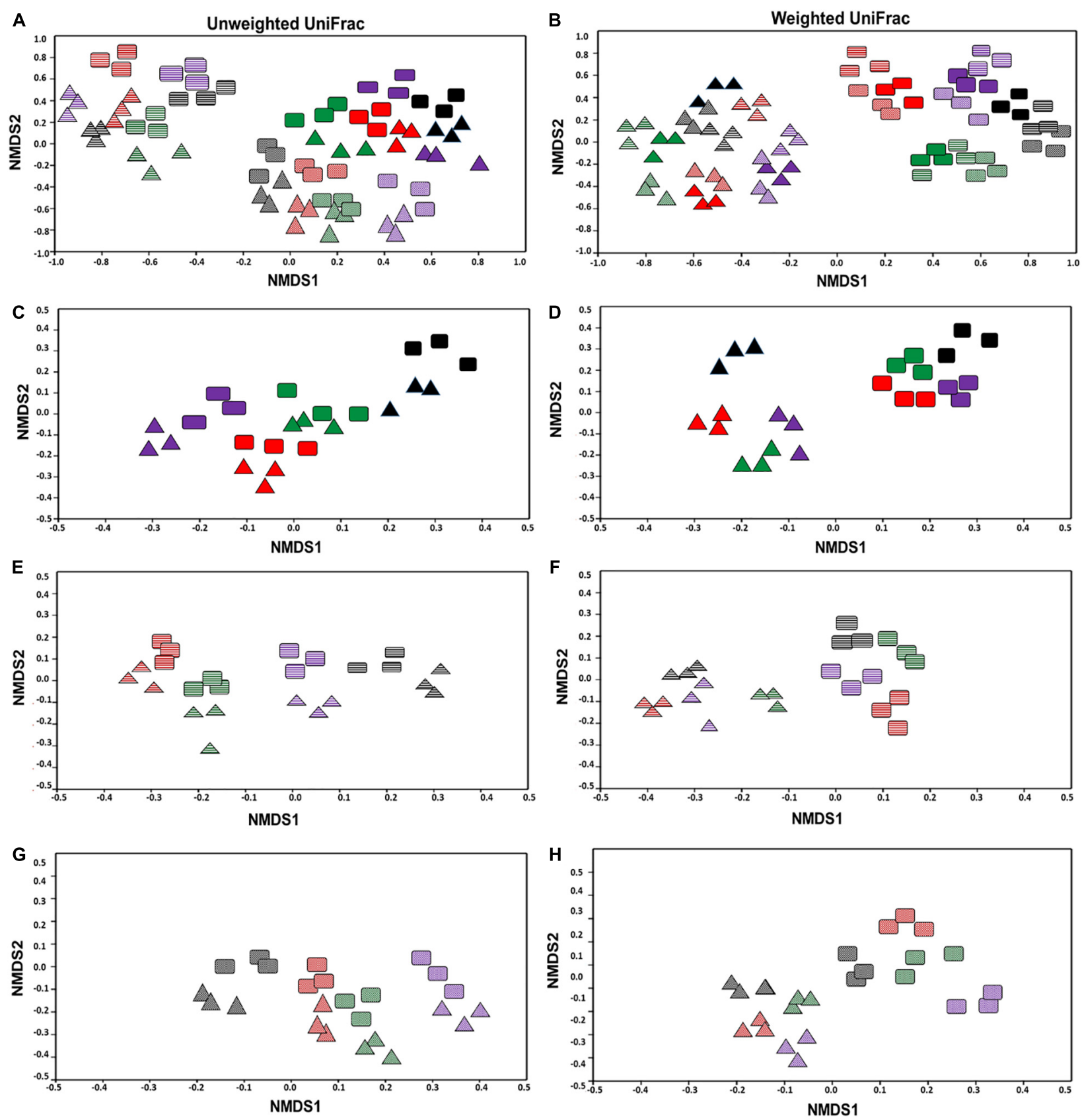

\begin{tabular}{|c|c|c|c|c|c|}
\hline \multirow[t]{2}{*}{ Sampling site } & \multirow[t]{2}{*}{ Sample type } & \multicolumn{4}{|c|}{ Plant species } \\
\hline & & Zygophyllum & \begin{tabular}{|l|} 
Haloxylon \\
\end{tabular} & Aerva & Capparis \\
\hline \multirow[t]{2}{*}{ Site 1} & Rhizosphere & & & & \\
\hline & \begin{tabular}{|l|} 
Root \\
endosphere
\end{tabular} & $\Delta$ & $\Lambda$ & $\Delta$ & $\Delta$ \\
\hline \multirow[t]{2}{*}{ Site 2} & Rhizosphere & 帮 & 㚸 & 司 & 荁 \\
\hline & \begin{tabular}{|l|} 
Root \\
endosphere
\end{tabular} & 点 & 点 & 点 & 査 \\
\hline \multirow[t]{2}{*}{ Site 3} & Rhizosphere & $\square$ & 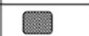 & $\square$ & 7 \\
\hline & \begin{tabular}{|l|} 
Root \\
endosphere
\end{tabular} & $\triangle$ & 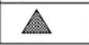 & $\triangle$ & $\Delta$ \\
\hline
\end{tabular}

FIGURE 3 | Beta diversity index of the bacterial communities in the rhizosphere and root endosphere of desert halophytes. Beta diversity was calculated by using weighted and unweighted UniFrac distances for non-metric multidimensional scaling plots (A,B) samples collected from all the sites, (C,D) samples collected from only site 1, (E,F) samples collected from only site 2, and (G,H) samples collected from only site 3 using unweighted (A,C,E,G) and weighted (B,D,F,H) UniFrac distances. 


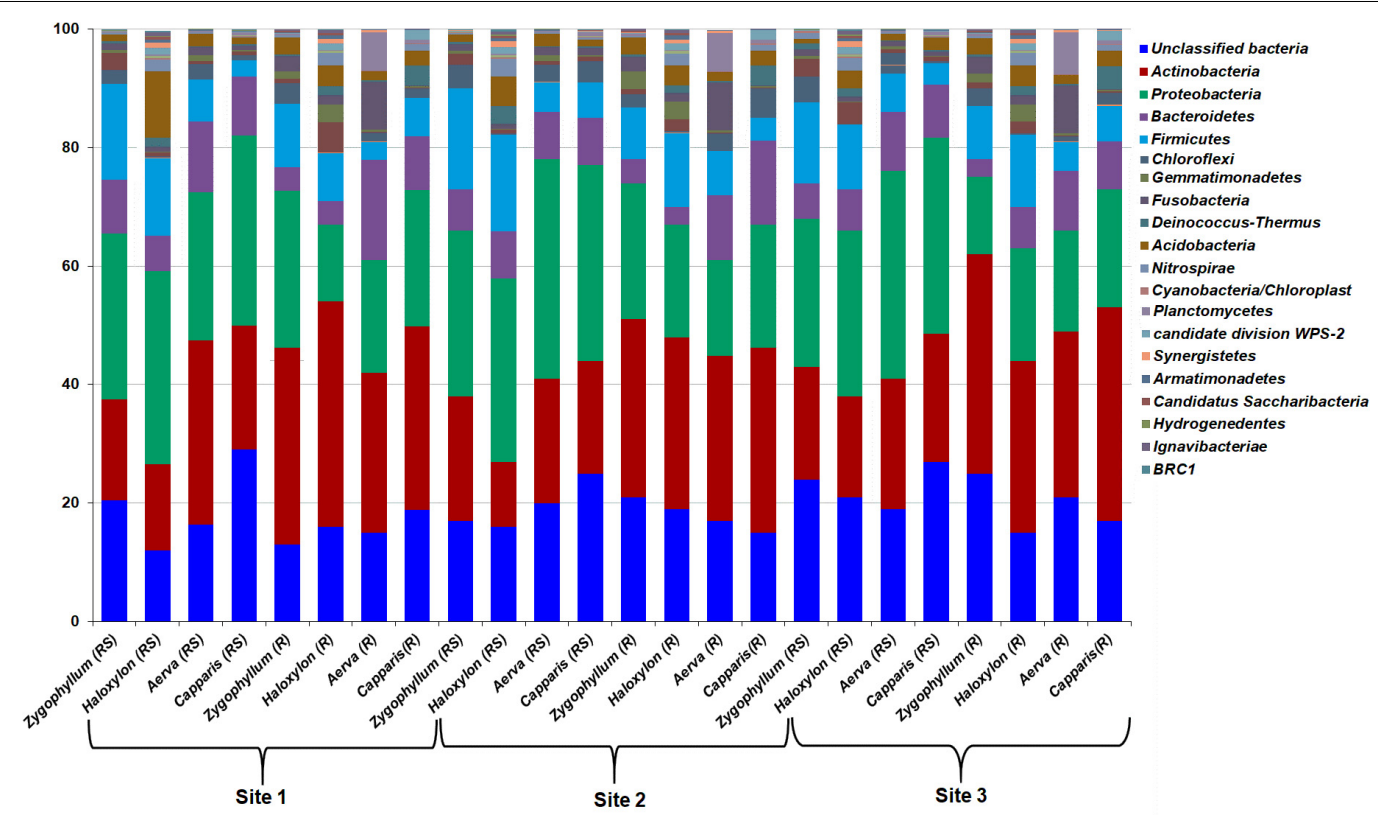

FIGURE 4 | Relative abundance of bacterial phyla from the rhizosphere (RS) and root endosphere (R) of desert halophytes collected from three sites of Cholistan.

\section{Microbial Diversity Comparisons at the Phylum and Class Level}

The OTUs from all the soil and root samples were assigned to 19 bacterial phyla. Microbial diversity at the phylum level showed significant differences among rhizosphere and root endosphere of halophytes across the three geographic sites (Figure 4). Proteobacteria (25.31-37.13\%) was the most abundant bacterial phylum identified from the rhizospheric soil samples of all plants while Actinobacteria (27.23-38.07\%) were dominant in root endosphere of all plants, across the three sites. Members of Firmicutes (8.51-17.19\%) were dominant in the rhizosphere and roots of Zygophyllum and Haloxylon whereas in case of Aerva and Capparis, Bacteroidetes (8.25-13.12\%) showed abundance across the three sites. Sequences related to Chloroflexi, Gemmatimonadetes, Fusobacteria, DeinococcusThermus, Acidobacteria, and Nitrospirae, were relatively less abundant, however, detected from all the rhizospheric soils with a significant difference in abundance across the three sites (Figure 4). At the phylum level, microbial communities associated with the rhizosphere and root endosphere of Haloxylon showed the highest variations as compared to microbial communities, identified from the rhizosphere and root endosphere of other desert halophytes, across the site 2. Our results also indicated that Actinobacteria significantly correlated with the roots and Proteobacteria significantly correlated with the rhizosphere of halophytes, whereas Nitrospirae and Synergistetes correlated least with the rhizosphere of halophytes, across the three sites.

The relative abundance of bacterial classes from the rhizosphere and roots of desert halophytes collected from the three geographic sites were compared in the form of a heatmap (Supplementary Figure 6).
Bacteroidia, Alphaproteobacteria, Bacilli, Betaproteobacteria, Sphingobacteria, Deltaproteobacteria, Gammaproteobacteria, Planctomycetia, Clostridia, and Halobacteria, were found to be abundant classes identified from all the rhizosphere and root samples of halophytes, collected from the site 1 (Supplementary Figure 6A). Bacilli, Alphaproteobacteria, Betaproteobacteria, Bacteroidia, Deltaproteobacteria, Flavobacteriia, Planctomycetia, Sphingobacteria, Acidobacteria_Gp7, Acidobacteria_Gp9, Nitrospira, and Halobacteria were dominating classes detected from all the rhizosphere and root samples of halophytes, collected from the site 2 (Supplementary Figure 6B). Bacilli, Alphaproteobacteria, Bacteroidia, Gammaproteobacteria, Deltaproteobacteria, Flavobacteriia, Planctomycetia, Acidobacteria_Gp9, Chlamydiia, Sphingobacteria, Nitrososphaeria, Acidobacteria_Gp7, Deinococci, Holophagae, and Halobacteria were dominating classes detected from all the rhizosphere and root samples of halophytes, collected from the site 3 (Supplementary Figure 6C). The cluster structure showed a different distribution pattern in rhizospheric soils as compared to root endosphere of halophytes, across the three sites. At class level also, the highest variations were observed between the rhizosphere and root endosphere of Haloxylon, collected from the geographic site 2 .

\section{Microbial Diversity Comparisons of Rhizosphere and Root Endosphere Across Three Geographic Sites at Genus Level}

The phylotypes identified from the rhizosphere and root endosphere of halophytes were compared across the three geographic sites. Venn diagram analysis revealed that a total 

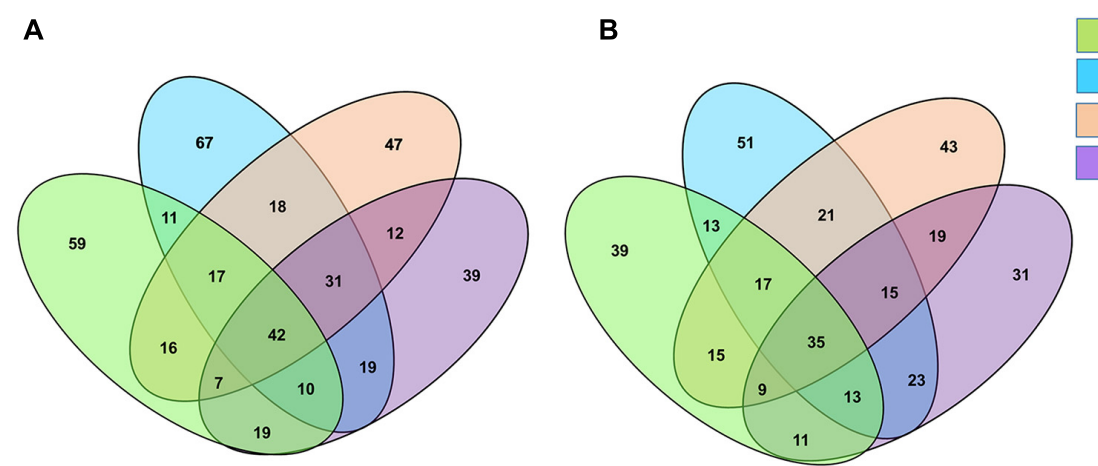

Zygophyllum simplex

Haloxylon salicoricum

Aerva javanica

Capparis decidua
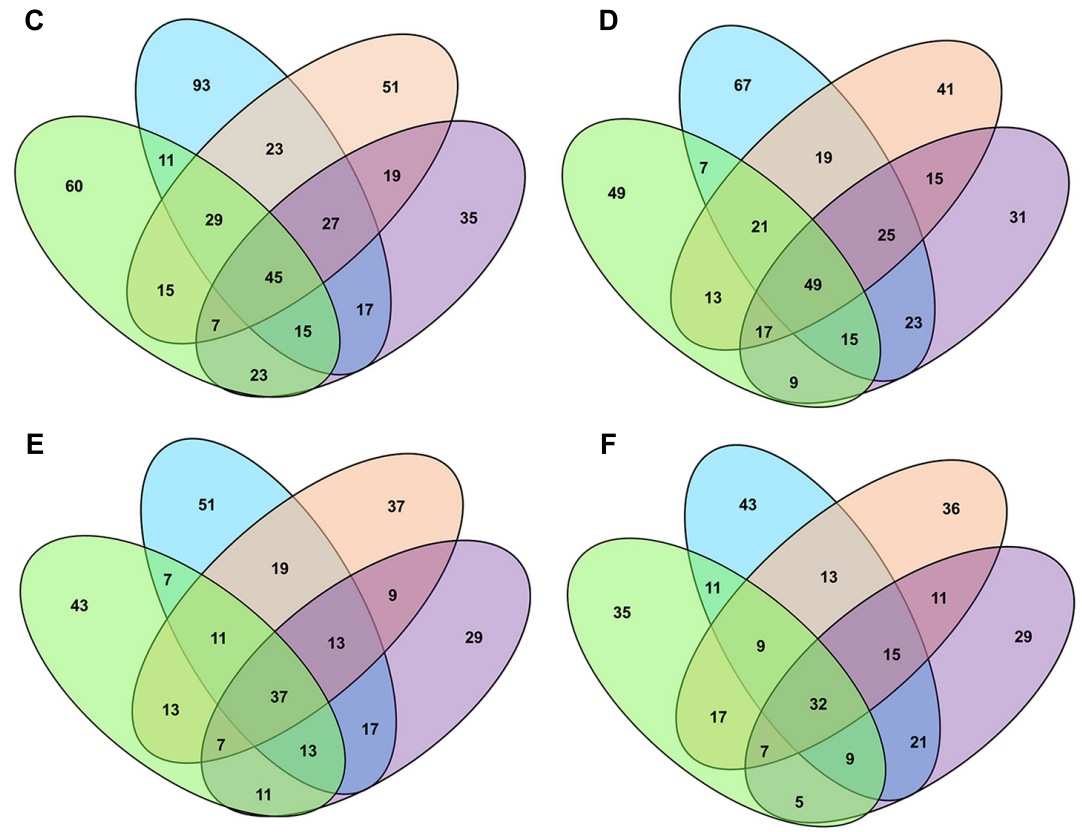

FIGURE $\mathbf{5}$ | Venn diagram showing the numbers of bacterial genera identified from the rhizosphere and the root endosphere of desert halophytes. (A,B) Samples collected from the site 1, (C,D) Samples collected from the site 2, and (E,F) Samples collected from the site 3.

of 765 phylotypes from the rhizosphere and 682 phylotypes from the root endosphere of halophytes have been identified from the site 1 (Figures 5A,B), 869 phylotypes from the rhizosphere and 790 phylotypes from the root endosphere of halophytes from the site 2 (Figures $\mathbf{5 C , D}$ ), and 592 phylotypes from the rhizosphere and 547 phylotypes from the root endosphere of halophytes have been identified from the site 3 (Figures 5E,F).

Overall, maximum microbial diversity was detected from the rhizosphere and root endosphere of all plants collected from the geographic site 2. A total of 45 phylotypes were commonly identified from all the rhizospheric soils and $60,93,51$, and 35 phylotypes were exclusively identified from the rhizosphere of Zygophyllum, Haloxylon, Aerva, and Capparis, respectively (Figure 5C). From the root endosphere of halophytes, 49 phylotypes were commonly identified and $49,67,41$, and 31 phylotypes were exclusive to the root endosphere associated microbial diversity of Zygophyllum, Haloxylon, Aerva, and Capparis, respectively (Figure 5D).
The results of LEfSe analysis showed the differences among the dominant bacterial genera from the rhizosphere and root endosphere of halophytes across all the sampling sites (Figure 6 and Supplementary Figure 7). The top five bacterial genera based on LDA scores were compared among the sample-type, plant species and across the sites. Bacterial genera, including Bacillus, Kocuria, Pseudomonas, Halomonas, and Flavobacterium were commonly identified from the rhizosphere and root endosphere of halophytes across all the geographic sites (Figure 6, Supplementary Figure 7 and Supplementary Tables 5-7). All the plant species showed great variations from one plant to another, however, within sample-type (the rhizosphere and root endosphere), there was no significant variation. Bacterial genera Virgibacillus, Oceanobacillus, and Planococcus (Firmicutes); Aeromonas, Marinobacter, Enterobacter, and Citrobacter (Proteobacteria); Kocuria, Solirubrobacter, Rubrobacter, Microbacterium, and Arthrobacter (Actinobacteria); were exclusively identified from the rhizosphere and root endosphere of Zygophyllum 


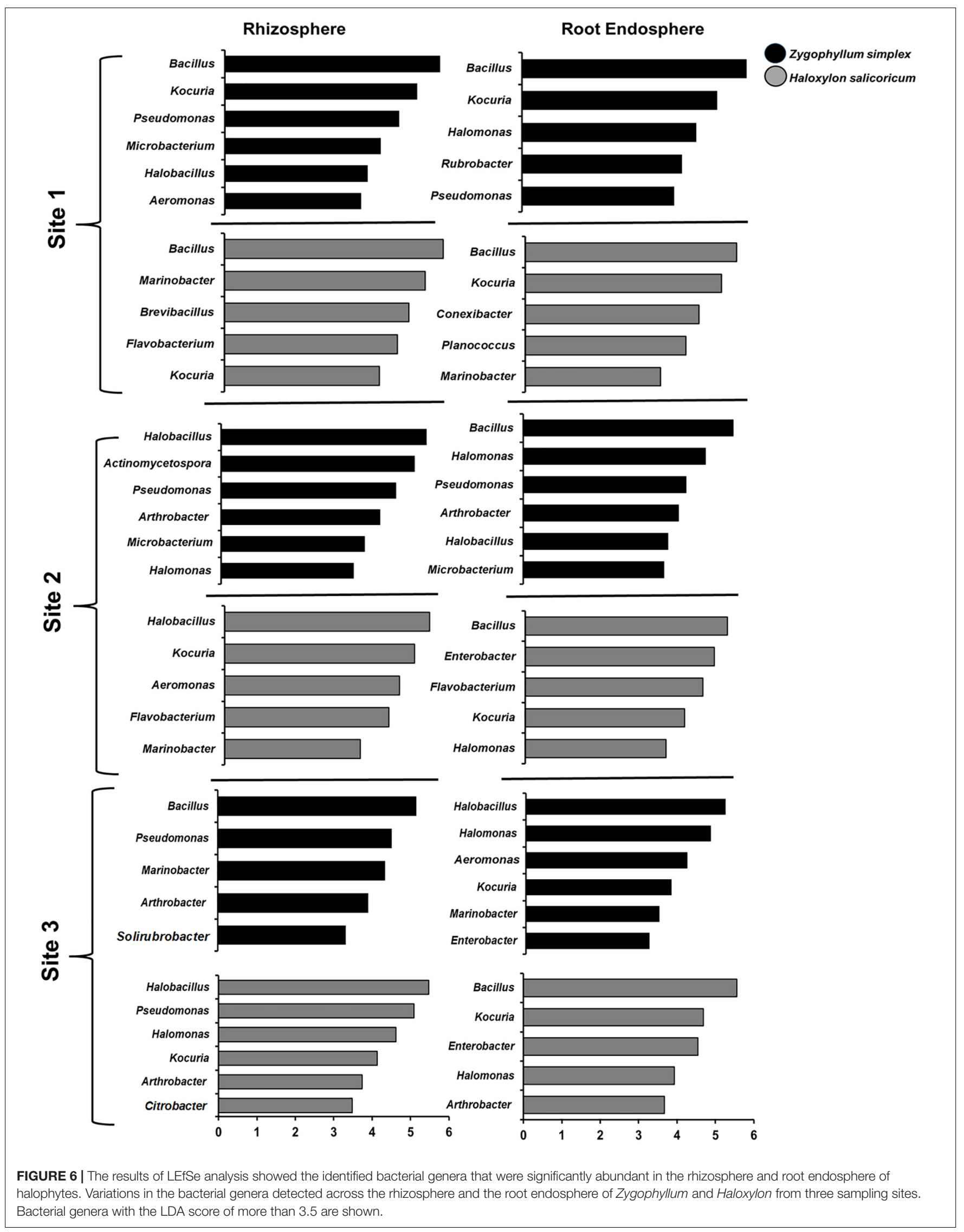



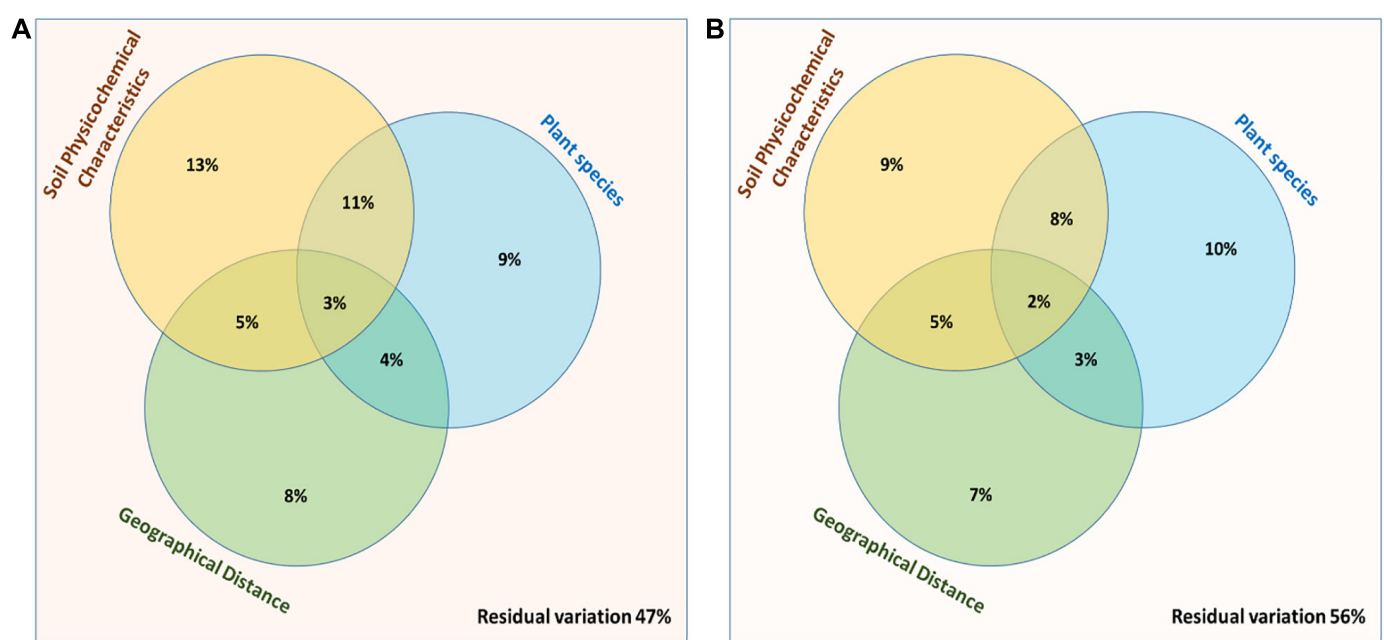

FIGURE 7 | Partitioning between the biological variations in the bacterial community structure. Three explanatory matrices were used here, containing variables pertaining to plant species, soil physicochemical characteristics and geographical distance on composition of (A) Rhizosphere and (B) Root endosphere microbiomes.

and Haloxylon while Burkholderia, Serratia, and Klebsiella (Proteobacteria), and Polaribacter (Bacteroidetes) were identified only from the rhizosphere and root endosphere of Aerva and Capparis. At the genus level, Haloxylon showed the greatest variations between the rhizosphere and root endosphere from the site 2 (Figure 6 and Supplementary Table 6). These results also confirmed the abundance of bacterial phyla Proteobacteria, Actinobacteria, Firmicutes, and Bacteroidetes, as the top bacterial genera from all the soil and root samples across all sampling sites.

\section{Factors Influencing Bacterial Community Structure and Distribution}

A multivariate variation partitioning approach was used to study the effects of soil physicochemical characteristics and the geographical distance on the overall variation of rhizosphere and root endosphere microbiomes based on the most abundant OTUs. A combination of environmental parameters; plant species, soil physicochemical characteristics, and geographical distance could explain $53 \%$ of their overall biological variations while $47 \%$ variations were unexplained in case of rhizosphere microbiomes (Figure 7A) while from the root endosphere microbiomes, different factors could explain only $44 \%$ of their overall biological variations while 56\% variations were unexplained (Figure 7B).

\section{Functional Profile of Bacterial Communities}

The results for the prediction of functional profiles of bacterial genera with specific plant growth promoting traits showed that nitrogen fixers were more abundant in the root endosphere of all the halophytes across the geographic sites 1 and 2 (Figures 8A,B). Mineral (Phosphate, potassium, and zinc) solubilizers and IAA (indole-3-acetic acid) producers were more dominant in the rhizosphere of Zygophyllum, Haloxylon, and
Aerva as compared to root endosphere across all sites. Bacterial strains with biocontrol activity were found to be most abundant in the rhizosphere of Haloxylon and Capparis, from the site 2 (Figures 8B,C). Microbes involved in bioremediation of toxic compounds were more abundant in the rhizosphere and root endosphere of Haloxylon, Aerva, and Capparis, across all sites. More than $83 \%$ bacterial strains identified in this study were halophilic and alkaliphilic in nature (Figures 8A,B).

\section{DISCUSSION}

Bacterial strains isolated from the extreme environments have special physiological and genetic characteristics to survive under such conditions. Cholistan desert is considered as naturally occurring extreme environment with drought, salinity and high temperature as the main abiotic factors. In this study, microbial diversity was compared from the rhizosphere and root endosphere of desert halophytes such as Zygophyllum simplex, Haloxylon salicoricum, Aerva javanica, and Capparis decidua, collected from three geographic sites of Cholistan desert, Pakistan by using $16 \mathrm{~S}$ rRNA based Illumina sequencing. The main objective of this study was to evaluate the impact of plant species, sample-type (rhizospheric and root endosphere) and geographic site, on the composition of microbial communities associated with desert halophytes. A number of studies have been previously reported on microbial diversity analyses from halophytes and xerophytes, such as microbial communities associated with the root endosphere of Halocnemum, Halostachys, Lycium, and Salicornia, using high throughput sequencing approaches (Coleman-Derr et al., 2016; Yuan et al., 2016; Tian and Zhang, 2017; Fitzpatrick et al., 2018). Here, we have reported the microbial diversity associated with the rhizosphere and root endosphere of halophytes, collected from Cholistan, Pakistan, by using culture-independent approaches. 


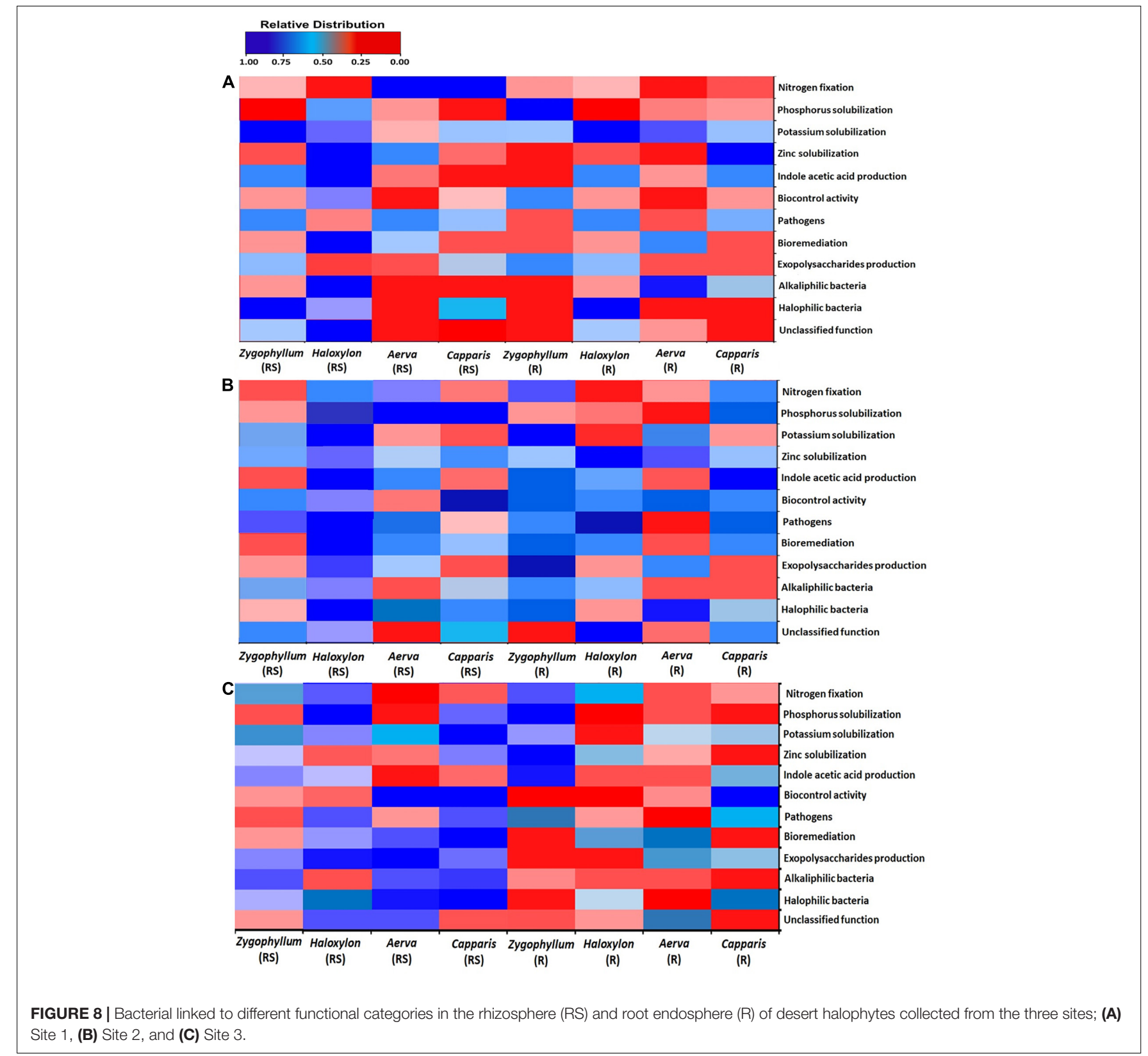

The rhizosphere and root endosphere associated microbial communities of a plant are affected by plant type, plant compartment and environmental factors such as geographic location, soil $\mathrm{pH}$, salinity, moisture content, soil leaching, erosion, and loss of certain nutrients (Pan et al., 2014; Fang et al., 2016; Mukhtar et al., 2018). Microbial diversity associated with the rhizosphere and root endosphere of Zygophyllum and Haloxylon showed a significant difference as compared to other halophytes, across all geographic sites. The results of sequence analysis of the 16S rRNA gene described that overall maximum microbial diversity identified from the geographic site 2 with more than $98.57 \%$ of retrieved sequences assigned to the domain Bacteria and $4.43 \%$ sequences related to unclassified microorganisms. Previous studies on
Cacti and Agave species also reported the similar pattern of microbial diversity (Coleman-Derr et al., 2016; Fonseca-Garcia et al., 2016). At the phylum level, microbial communities identified from the rhizosphere of halophytes showed more diversity as compared to those associated with the root endosphere, across all the three sites. Among the plant species, Haloxylon showed overall more microbial diversity as compared to other halophytes, used in this study. Bacterial phyla Actinobacteria, Proteobacteria, Bacteroidetes, Firmicutes, Acidobacteria, Chloroflexi, Planctomycetes, and Fusobacteria were commonly identified from all rhizosphere and root samples. Some previous studies showed that the abundance of certain phyla, such as Actinobacteria, Proteobacteria, Firmicutes, and CRC1 increased in the rhizosphere of desert halophytes 
such as Halostachys caspica, and Salicornia alterniflora, with the increase in soil salinity and moisture content (Marasco et al., 2016). Increase in soil salinity also negatively affect the abundance of certain phyla, such as Chloroflexi, Acidobacteria, Nitrospirae, and Synergistetes (Foesel et al., 2014; Yan et al., 2015; Mukhtar et al., 2018).

Overall, Actinobacteria were found to be more abundant in the root endosphere of halophytes than the rhizosphere across all the geographic sites, but within plant species, Zygophyllum and Haloxylon showed more abundance of Actinobacteria across the geographic site 1 and 3 . The results of $16 \mathrm{~S}$ rRNA based metagenomic analysis showed that bacterial genera Kocuria, Brevibacterium, Micrococcus, Streptomyces, Solirubrobacter, and Nocardia (Actinobacteria) were identified from the root endosphere of all plants. Previous studies also showed that Actinobacteria are dominant in the salinity and drought affected soils. These bacteria have the potential ability to promote plant growth, to produce a large number of antimicrobial compounds and to degrade a variety of toxic organic compounds from the polluted saline environments (Dupont et al., 2014; Deng et al., 2015; Zhang et al., 2015). Bacterial genera Asanoa, Actinoplanes, Marmoricola, and Microlunatus were detected only from the rhizosphere of Aerva and Capparis from the geographic site 1 and 2 . These strains have previously been isolated from the rhizosphere of halophytes such as Phragmites australis, Salsola stocksii, and Atriplex amnicola. These can be used for biofuel production and play an important role in carbon cycling of soil ecosystems (Borruso et al., 2014; Krivushin et al., 2015; Mukhtar et al., 2018).

This study showed that the sequences related to Proteobacteria (Alphaproteobacteria, Betaproteobacteria, and Gammaproteobacteria) were detected from the rhizosphere and root endosphere microbiomes of all the plants but with more abundance in the rhizosphere samples. Bacterial genera Pseudomonas, Halomonas, Enterobacter, Burkholderia, Azospirillum, Marinobacter, and Geobacter were dominant as compared to other strains in all the rhizosphere samples of all the plants, as compared to root samples across the site 1 and 3 while Serratia, Azotobacter, and Xanthomonas were identified only from the rhizosphere of Aerva and Capparis, collected from the geographic site 2. Previous studies also reported the isolation and identification of these Protobacterial genera from a number of saline and arid environments (Jiang et al., 2014; Mukhtar et al., 2018, 2020). These bacteria can be used as biofertilizers, as biocontrol agents and for bioremediation of a variety of hazardous compounds from polluted saline and arid environments (Mukhtar et al., 2017a).

Members of Firmicutes were more abundant in the rhizosphere and root endosphere of Zygophyllum and Haloxylon as compared to Capparis and Aerva across all the three sites. As expected from the previous studies, these results also confirmed the correlation of increase in soil salinity and abundance of Firmicutes. Bacillus, Halobacillus, Virgibacillus, Oceanobacillus, Marinococcus, Planococcus, and Exiguobacterium were dominant genera detected in all the rhizosphere and root endosphere of halophytes. Within plant species, Haloxylon showed the maximum diversity of Firmicutes at the genus level between the rhizosphere and root endosphere from the geographic site 2. Bacillus strains have plant growth promoting and biocontrol abilities and can be used as potential biofertilizers (Krid et al., 2010; Mukhtar et al., 2017a). Bacillus like bacteria isolated and characterized from saline environments have novel halophilic enzymes which can be used for a number of industries and bioremediation of organic pollutants in arid and saline environments (Oren, 2015; Liu et al., 2017; Mukhtar et al., 2017b, 2019).

Dominant genera from the phylum Bacteroidetes identified in this study were Flavobacterium, Salinibacter, Sphingobacterium, and Cytophaga. These genera were detected from the rhizosphere and root endosphere of all the plants across the three geographic sites. Polaribacter, Chlorobium, and Chitinivibrio were exclusively identified from the rhizosphere and root endosphere of Aerva and Capparis. Members of Bacteroidetes are usually found to be dominant in the rhizosphere of plants growing under salinity and drought stress conditions (Coleman-Derr et al., 2016; Gibtan et al., 2017). Gp4, Gp6, Gp7, Gp10, and Gp21 (Acidobacteria) were detected in this study. Acidobacteria were more abundant in the rhizosphere as compared to root endosphere of all the halophytes across the site 1 and 3. A number of previous studies also reported the abundance of Acidobacteria in the rhizosphere of halophytes and other hypersaline environments (Zhang et al., 2015; Mukhtar et al., 2016). Sequences related to bacterial phyla Fusobacteria, Gemmatimonadetes, Chloroflexi, Synergistetes, Planctomycetes, Deinococcus-Thermus, Armatimonadetes, and Nitrospirae were identified in the rhizosphere of all the halophytes but they were relatively less abundant across the sites 1 and 3 as compared to the site 2 . These phyla have already been detected in various extreme environments such as rhizosphere of halophytes and xerophytes, marine water, rock sediments, anaerobic sludge, and hot spring water (Iverson et al., 2012; Zenga et al., 2014).

\section{CONCLUSION}

This study described the microbial diversity analysis of the rhizosphere and root endosphere of desert halophytes, collected from the three geographic sites of Cholistan, Pakistan by using 16S rRNA based Illumina sequencing. The present study reveals that plant species, plant compartments and a change in soil physicochemical characteristics affect the composition of rhizosphere and root endophytic microbial communities. Phylogenetic profiling showed that bacterial phyla Actinobacteria, Proteobacteria, Bacteroidetes, Firmicutes, and Fusobacteria were commonly identified from all the rhizosphere and root samples with significant variations between the rhizosphere and root endosphere, plant species across the three sites. Microbial diversity associated with the rhizosphere and root endosphere of Haloxylon collected from the geographic site 2 showed the greatest variations at the phylum and genus level. From the rhizosphere and the roots of each plant, certain bacterial genera were more dominant. More than $80 \%$ bacterial genera identified in this study have the potential ability to promote plant growth under salinity and drought stress environments and could be used as potential candidates for biofertilizers in salt affected agricultural lands. 


\section{DATA AVAILABILITY STATEMENT}

The datasets presented in this study can be found in online repositories. The names of the repository/repositories and accession number(s) can be found below: https://www.ncbi.nlm. nih.gov/, SRR9588854-SRR9588861.

\section{AUTHOR CONTRIBUTIONS}

SMu: conducted the experiment, analyzed the data, and prepared the manuscript. SMe: guided in experiment plan and edited the manuscript. KM: supervised the research and edited the manuscript. All authors contributed to the article and approved the submitted version.

\section{REFERENCES}

Abhauer, K. P., Wemheuer, B., Daniel, R., and Meinicke, P. (2015). Tax4Fun: predicting functional profiles from metagenomic 16S rRNA data. Bioinformatics. 31, 2882-2884. doi: 10.1093/bioinformatics/btv287

Adviento-Borbe, M. A., Doran, J. W., Drijber, R. A., and Dobermann, A. (2006). Soil electrical conductivity and water content affect nitrous oxide and carbon dioxide emissions in intensively managed soils. J. Environ. Qual. 35, 1999-2010. doi: $10.2134 /$ jeq2006.0109

Anderson, J. M., and Ingram, J. S. (1993). Tropical Soil Biology and Fertility: A Handbook of Methods, 2nd Edn. Wallingford: CAB International, 93-94.

Borruso, L., Bacci, G., Mengoni, A., De Philippis, R., and Brusetti, L. (2014). Rhizosphere effect and salinity competing to shape microbial communities in Phragmites australis (Cav.) Trin. exSteud. FEMS Microbiol. Lett. 359, 193-200. doi: 10.1111/1574-6968.12565

Bulgarelli, D., Schlaeppi, K., Spaepen, S., Themaat, E., and Schulze-Lefert, P. (2013). Structure and functions of the bacterial microbiota of plants. Ann. Rev. Plant Biol. 64, 807-838. doi: 10.1146/annurev-arplant-050312-120106

Caporaso, J. G., Kuczynski, J., Stombaugh, J., Bittinger, K., et al. (2010). QIIME allows analysis of high throughput community sequencing data. Nat. Meth. 7, 335-336.

Castillo-Carvajal, L. C., Sanz-Martín, J. L., and Barragán-Huerta, B. E. (2014). Biodegradation of organic pollutants in saline wastewater by halophilic microorganisms: a review. Environ. Sci. Pollut. Res. Int. 21, 9578-9588. doi: 10.1007/s11356-014-3036-z

Chaudhary, D. R., Gautam, R. K., Yousuf, B., Mishra, A., and Jha, B. (2015). Nutrients, microbial community structure and functional gene abundance of rhizosphere and bulk soils of halophytes. Appl. Soil Ecol. 91, 16-26. doi: 10. 1016/j.apsoil.2015.02.003

Chaudhry, M. S., and Nasim, F. H. (1995). Combating desertification in Cholistan desert. Sci Tech Islamic World. 13, 75-85.

Coleman-Derr, D., Desgarennes, D., Fonseca-Garcia, C., Gross, S., Clingenpeel, S., Woyke, T., et al. (2016). Plant compartment and biogeography affect microbiome composition in cultivated and native Agave species. New Phytol. 209, 798-811. doi: 10.1111/nph.13697

Dagla, H. R., and Shekhawat, N. S. (2005). In vitro multiplication of Haloxylon recurvum (Moq.)- aplant for saline soil reclamation. J. Plant Biol. 7, 155-160.

Deng, S., Chang, X., Zhang, Y., Ren, L., Jiang, F., Qu, Z., et al. (2015). Nocardioides antarcticus sp. nov., isolated from marine sediment of Ardley cove. Int. J. Syst. Evol. Microbiol. 65, 2615-2621. doi: 10.1099/ijs.0.000309

DeSantis, T. Z., Hugenholtz, P., Larsen, N., Rojas, M., Brodie, E. L., Keller, K., et al. (2006). Green genes, a chimera checked 16S rRNA gene database and workbench compatible with ARB. Appl. Environ. Microbiol. 72, 5069-5072. doi: 10.1128/aem.03006-05

Dupont, C. L., Larsson, J., Yooseph, S., Ininbergs, K., Goll, J., et al. (2014). Functional tradeoffs underpin salinity-driven divergence in microbial community composition. PLoS One. 9:e89549. doi: 10.1371/journal.pone. 0089549

\section{ACKNOWLEDGMENTS}

We are highly thankful to Higher Education Commission [Project \# HEC (FD/2012/1843)] for research grant. We would like to express our gratitude to Dr. Samia Ahmad (Michigan State University) for her help in data analysis and Mr. Mukhtar Ahmad (Assistant Professor), Dyal Singh College, Lahore, for assistance in statistical analyses.

\section{SUPPLEMENTARY MATERIAL}

The Supplementary Material for this article can be found online at: https://www.frontiersin.org/articles/10.3389/fmicb. 2021.618742/full\#supplementary-material

English, J. P., and Colmer, T. D. (2011). Salinity and waterlogging tolerances in three stem- succulent halophytes (Tecticornia species) from the margins of ephemeral salt lakes. Plant Soil. 348, 379-396. doi: 10.1007/s11104-0110924-6

Etesami, H., and Beattie, G. A. (2018). "Plant-microbe interactions in adaptation of agricultural crops to abiotic stress conditions," in Probiotics and Plant Health, eds V. Kumar, M. Kumar, S. Sharma, and R. Prasad (Singapore: Springer), $163-200$.

Etesami, H., and Maheshwari, D. K. (2018). Use of plant growth promoting rhizobacteria (PGPRs) with multiple plant growth promoting traits in stress agriculture: action mechanisms and future prospects. Ecotoxicol. Environ. Saf. 156, 225-246. doi: 10.1016/j.ecoenv.2018.03.013

Fahmy, T. (2003). XLSTAT-Pro 7.0 (XLSTAT), Addinsoft. Paris: XLSTAT.

Fang, T. T., Pan, R. S., Jiang, J., He, F., and Wang, H. (2016). Effect of salinity on community structure and naphthalene dioxygenase gene diversity of a halophilic bacterial consortium. Front. Env. Sci. Eng. 10:16. doi: 10.1007/ s11783-016-0888-0

Fitzpatrick, C. R., Copeland, J., Wang, P. W., Guttman, D. S., Kotanen, P. M., and Johnson, M. T. J. (2018). Assembly and ecological function of the root microbiome across angiosperm plant species. Proc. Natl. Acad. Sci. 115, E1157E1165.

Foesel, B. U., Nägele, V., and Naether, A. (2014). Determinants of acidobacteria activity inferred from the relative abundances of $16 \mathrm{~S}$ rRNA transcripts in german grassland and forest soils. Environ. Microbiol. 16, 658-675. doi: 10. $1111 / 1462-2920.12162$

Fonseca-Garcia, C., Coleman-Derr, D., Garrido, E., Visel, A., Tringe, S. G., and Partida-Martínez, L. P. (2016). The cacti microbiome: interplay between habitat-filtering and host-specificity. Front. Microbiol. 7:150. doi: 10.3389/ fmicb.2016.00150

Gibtan, A., Park, K., Woo, M., Shin, J. K., Lee, D. W., et al. (2017). Diversity of extremely halophilic archaeal and bacterial communities from commercial salts. Front. Microbiol. 8:799. doi: 10.3389/fmicb.2017.00799

Good, I. J. (1953). The population frequencies of species and the estimation of population parameters. Biometrica. 40, 237-264. doi: 10.1093/biomet/40.3-4. 237

Herlemann, D. P., Labrenz, M., Jürgens, K., Bertilsson, S., Waniek, J. J., et al. (2011). Transitions in bacterial communities along the $2000 \mathrm{~km}$ salinity gradient of the Baltic Sea. ISME J. 5, 1571-1579. doi: 10.1038/ismej.2011.41

Hussain, A., Arshad, M., Zahir, Z. A., and Asghar, M. (2015). Prospects of zinc solubilizing bacteria for enhancing growth of maize. Pak J Agri Sci. 52, 915-922.

Iverson, V., Morris, R. M., Frazar, C. D., Berthiaume, C. T., Morales, R. L., and Armbrust, E. V. (2012). Untangling genomes from metagenomes: revealing an uncultured class of marine Euryarchaeota. Science 335, 587-590. doi: 10.1126/ science. 1212665

Jiang, J. Q., Pan, Y. Y., Hu, S. X., Zhang, X. X., Hu, B. Z., et al. (2014). Halomonas songnenensis sp nov., a moderately Halophilic bacterium isolated from saline and alkaline soils. Int. J. Syst. Evol. Microbiol. 64, 1662-1669. doi: 10.1099/ijs.0. 056499-0 
Koranda, M., Schnecker, J., Kaiser, C., Fuchslueger, L., Kitzler, B., Stange, C. F., et al. (2011). Microbial processes and community composition in the rhizosphere of European beech - the influence of plant C exudates. Soil Biol. Biochem. 43, 551-558. doi: 10.1016/j.soilbio.2010.11.022

Krid, S., Rhouma, A., Mogou, I., Quesada, J. M., Nesme, X., and Gargouri, A. (2010). Pseudomonas savastonoi endophytic bacteria in olive tree knots and antagonistic potential of strains of Pseudomonas fluorescens and Bacillus subtilis. J. Plant Pathol. 92, 335-341.

Krivushin, K., Kondrashov, F., Shmakova, L., Tutukina, M., Petrovskaya, L., and Rivkinaa, E. (2015). Two metagenomes from late pleistocene northeast siberian permafrost. Genome. Announc. 3, 1380-1400.

Kumar, S., Karan, R., Kapoor, S., Singh, S. P., and Khare, S. K. (2012). Screening and isolation of Halophilic bacteria producing industrially important enzymes. Braz. J. Microbiol. 43, 1595-1603. doi: 10.1590/s1517-83822012000400044

Liszka, M., Clark, M., Schneider, E., and Clark, D. S. (2012). Nature versus nurture: developing enzymes that function under extreme conditions. Ann. Rev. Chem. Biomol. Eng. 3, 77-102. doi: 10.1146/annurev-chembioeng-061010-114239

Liu, M., Cui, Y., Chen, Y., Lin, X., Huang, H., and Bao, S. (2017). Diversity of Bacillus-like bacterial community in the sediments of the Bamenwan mangrove wetland in Hainan, China. Can. J. Microbiol. 63, 238-245. doi: 10.1139/cjm2016-0449

Lozupone, C., Lladser, M. E., Knights, D., Stombaugh, J., and Knight, R. (2011). UniFrac: an effective distance metric for microbial community comparison. ISME J. 5, 169-172. doi: 10.1038/ismej.2010.133

Marasco, R., Mosqueira, M. J., Fusi, M., Ramond, J. B., Merlino, G., Booth, J. M., et al. (2016). Salicornia strobilacea (synonym of Halocnemum strobilaceum) grown under different tidal regimes selects rhizosphere bacteria capable of promoting plant growth. Front. Microbiol. 7:1286. doi: 10.3389/fmicb.2016. 01286

Mukhtar, S., Mehnaz, S., Mirza, M. S., and Malik, K. A. (2019). Isolation and characterization of halophilic bacteria from the rhizosphere of halophytes and non-rhizospheric soil samples. Braz. J. Microbiol. 50, 85-97.

Mukhtar, S., Mirza, B. S., Mehnaz, S., Mirza, M. S., Mclean, J., and Malik, K. A. (2018). Impact of soil salinity on the structure and composition of rhizosphere microbiome. World J. Microbiol. Biotech. 34:136.

Mukhtar, S., Mirza, M. S., Awan, H. A., Maqbool, A., Mehnaz, S., and Malik, K. A. (2016). Microbial diversity and metagenomic analysis of the rhizosphere of Para Grass (Urochloa mutica) growing under saline conditions. Pak. J. Bot. 48, 779-791.

Mukhtar, S., Ishaq, A., Hassan, S., Mehnaz, S., Mirza, M. S., and Malik, K. A. (2017a). Comparison of microbial communities associated with halophyte (Salsola stocksii) and non-halophyte (Triticum aestivum) using cultureindependent approaches. Pol. J. Microbiol. 66, 375-386.

Mukhtar, S., Shahid, I., Mehnaz, S., and Malik, K. A. (2017b). Assessment of two carrier materials for phosphate solubilizing biofertilizers and their effect on growth of wheat (Triticum aestivum). Microbiol. Res. 205, 107-117. doi: 10.1016/j.micres.2017.08.011

Mukhtar, S., Zareen, M., Khaliq, Z., Mehnaz, S., and Malik, K. A. (2020). Phylogenetic analysis of halophyte-associated rhizobacteria and effect of halotolerant and halophilic phosphate-solubilizing biofertilizers on maize growth under salinity stress conditions. J. Appl. Microbiol. 128, 556-573. doi: 10.1111/jam.14497

Olsen, S. R., Cole, C. V., Watanabe, F. S., and Dean, L. A. (1954). Estimation of Available Phosphorus in Soils by Extraction with Sodium Bicarbonate. Washington D.C: USDA Circular, Printing Office,

Oren, A. (2013). Life at high salt concentrations, intracellular $\mathrm{KCl}$ concentrations, and acidic proteomes. Front. Microbiol. 4:315. doi: 10.3389/fmicb.2013.00315

Oren, A. (2015). Halophilic microbial communities and their environments. Curr. Opin. Microbiol. 33, 119-124. doi: 10.1016/j.copbio.2015.02.005

Pan, Y., Cassman, N., Hollander, M., Mendes, L. W., Korevaar, H., et al. (2014). Impact of long-term N, P, K, and NPK fertilization on the composition and potential functions of the bacterial community in grassland soil. FEMS Microbiol. Ecol. 90, 195-205. doi: 10.1111/1574-6941.12384

Ramadoss, D., Lakkineni, V. K., Bose, P., Ali, S., and Annapurna, K. (2013). Mitigation of salt stress in wheat seedlings by halotolerant bacteria isolated from saline habitats. Springerplus. 2:6. doi: 10.1186/2193-1801-2-6

Rengasamy, R. (2006). World salinization with emphasis on Australia. J. Exp. Bot. 57, 1017-1023. doi: 10.1093/jxb/erj108

Santhanam, R., Oh, Y., Kumar, R., Weinhold, A., Luu, V. T., Groten, K., et al. (2017). Specificity of root microbiomes in native-grown Nicotiana attenuata and plant responses to UVB increase Deinococcus colonization. Mol. Ecol. 26, 2543-2562. doi: 10.1111/mec.14049

Segata, N., Izard, J., Waldron, L., Gevers, D., Miropolsky, L., Garrett, W., et al. (2011). Metagenomic biomarker discovery and explanation. Gen. Biol. 12, R60.

Tian, X. Y., and Zhang, C. S. (2017). Illumina-based analysis of endophytic and rhizosphere bacterial diversity of the coastal halophyte Messerschmidia sibirica. Front. Microbiol. 8:2288. doi: 10.3389/fmicb.2017.02288

Tian, Y. Q., and Gao, L. H. (2014). Bacterial diversity in the rhizosphere of cucumbers grown in soils covering a wide range of cucumber cropping histories and environmental conditions. Microbial. Ecol. 68, 794-806. doi: 10.1007/ s00248-014-0461-y

Vogel, H. (1978). Vogel's Textbook of Quantitative Inorganic Analysis, 4th Edn. London: Longman.

Walkley, A., and Black, I. A. (1934). An examination of degtjareff method for determining soil organic matter and a proposed modification of the chromic acid titration method. Soil Sci. 37, 29-37. doi: 10.1097/00010694-19340100000003

Wang, Q., Garrity, G. M., Tiedje, J. M., and Cole, J. R. (2007). Naive Bayesian classifier for rapid assignment of rRNA sequences into the new bacterial taxonomy. Appl. Environ. Microbiol. 73, 5261-5267. doi: 10.1128/aem.00 062-07

Yan, N., Marschner, P., Cao, W., Zuo, C., and Qin, W. (2015). Influence of salinity and water content on soil microorganisms. ISWCR 3:316323.

Yuan, Z., Druzhinina, I. S., Labbé, J., Redman, R., Qin, Y., Rodriguez, R., et al. (2016). Specialized microbiome of a halophyte and its role in helping non-host plants to withstand salinity. Sci. Rep. 6:32467.

Zenga, Y., Fengb, F., Medováa, H., Deana, J., and Koblížeka, M. (2014). Functional type 2 photosynthetic reaction centers found in the rare bacterial phylum Gemmatimonadetes. PNAS. 111, 7795-7800. doi: 10.1073/pnas.1400295111

Zhang, H., Wang, Z., Zhang, Y., Ding, M., and Li, L. (2015). Identification of trafficrelated metals and the effects of different environments on their enrichment in roadside soils along the Qinghai-Tibet highway. Sci. Total Environ. 522, 160-172. doi: 10.1016/j.scitotenv.2015.03.054

Zhao, S., Zhou, N., Zhao, Z. Y., Zhang, K., Wu, G. H., and Tian, C. Y. (2016). Isolation of endophytic plant growth-promoting bacteria associated with the halophyte Salicornia europaea and evaluation of their promoting activity under salt stress. Curr. Microbiol. 73, 574-581. doi: 10.1007/s00284-0161096-7

Zhou, N., Zhao, S., and Tian, C. Y. (2017). Effect of halotolerant rhizobacteria isolated from halophytes on the growth of sugar beet (Beta vulgaris L.) under salt stress. FEMS Microbiol. Lett. 364:fnx091.

Conflict of Interest: The authors declare that the research was conducted in the absence of any commercial or financial relationships that could be construed as a potential conflict of interest.

Copyright (c) 2021 Mukhtar, Mehnaz and Malik. This is an open-access article distributed under the terms of the Creative Commons Attribution License (CC BY). The use, distribution or reproduction in other forums is permitted, provided the original author(s) and the copyright owner(s) are credited and that the original publication in this journal is cited, in accordance with accepted academic practice. No use, distribution or reproduction is permitted which does not comply with these terms. 